\title{
Editorial: More Answers About Prophylactic Mastectomy
}

\author{
William C. Wood, MD \\ Department of Surgery, Emory University School of Medicine, Winship Cancer Institute, 1364 Clifton Road, \\ NE, Atlanta, GA 30322
}

Heemskerk-Gerritsen et al. ${ }^{1}$ continue to add to our understanding of the surgical management of hereditary breast and ovarian cancer. In their fine article, they contribute to our knowledge of control, evaluation, staging, and reconstruction.

\section{CONTROL}

The Rotterdam series is reassuring in demonstrating that bilateral prophylactic mastectomy will almost always prevent the development of breast cancer. In addition to the 145 women with defined BRCA1 or two mutations followed for a median of 4.5 years, there were 32 at risk due to history, 91 with defined risk presenting with breast cancer, and 90 at risk by history presenting with breast cancer. This large collection adds to other studies and provides further evidence that such prophylactic surgery for hereditary breast and ovarian cancer radically reduces both the risk and anxiety, although eliminates neither completely.

\section{EVALUATION}

The importance of careful evaluation preceding prophylactic surgery, stressed by the authors, has been made easier by the increasing availability of radiologists skilled in the interpretation of MRIs of the breast. The increased sensitivity of the technique

Received May 30, 2007; accepted May 31, 2007; published online: September 25, 2007.

Address correspondence and reprint requests to: William $\mathrm{C}$. Wood, MD; E-mail: william.wood@emory.edu

Published by Springer Science+Business Media, LLC $\odot 2007$ The Society of Surgical Oncology, Inc. was initially undermined by a lack of specificity. With growing experience, the specificity of MRI detection has reached greater than $90 \%$, similar to that achieved with mammography. However, in Dr. Christiane Kuhl's series, using mammography in BRCA mutation carriers, only one-third of their cancers were detected, whereas MRI identified over $90 \%{ }^{2}$ This prevents the unhappy experience of finding that surgery directed to prevention was actually being performed as therapy.

\section{STAGING}

With careful evaluation preceding prophylactic mastectomy there is no need for the routine inclusion of sentinel lymph node biopsy. The authors' finding of less than $1 \%$ incidence of unanticipated invasive cancer answers this question that has enjoyed debate in breast evaluation rounds in recent years.

\section{RECONSTRUCTION}

The authors concluded their evaluation of reconstruction with this sentence: "In this respect, patients should be informed preoperatively that an optimal cosmetic effect cannot unconditionally be achieved in just one single operation." Who could find fault with that? They react to a concern that reconstruction may be presented as restoration of the breasts as originally formed. While some patients may find that their reconstructed breasts are closer to a common ideal than their native breasts, many who began with near perfection are surprised that it takes additional tweaking after initial reconstruction to achieve something that they can admire. Setting appropriate 
expectations is a task for the entire team. Many of the complications listed by the authors, however, are true surgical complications, not failures of aesthetic perfection. The team also needs to monitor these and assure that any complication offers opportunity for improvement in technique.

Perhaps because the focus of the authors' publication is on breast cancer, the emphasis on the greater danger of ovarian cancer seems a secondary consideration. With breast MRI screening, we are often able to detect breast cancer at a minimal stage in these women. We are still unable to detect ovarian cancer in most instances until it has progressed to an advanced stage. By turning attention to counseling women at hereditary risk of ovarian cancer, a different algorithm can emerge. Very young mutation carriers can use birth control pills for 5 years, with a halving of their risk of ovarian cancer. As soon as they have had their families, salpingo-oophorectomy can not only greatly reduce the risk of ovarian cancer, but breast cancer risk is reduced by nearly two-thirds if this procedure is performed prior to age 40 and roughly halved if performed over 40 years of age. ${ }^{3}$ This reduction can be improved by the use of tamoxifen. The magnitude of the tamoxifen benefit is a surprising $50 \%$ reduction in risk when used by itself. When used following oophorectomy it appears to provide an additional $17 \%$ reduction. ${ }^{4}$ Greater numbers of patients so treated will be required for more definitive statistics. It is apparent, however, that attention to preventing the more insidious ovarian cancer by oophorectomy dramatically reduces the breast cancer risk.

When a woman realizes that the $80 \%$ lifetime risk number that she has heard can be reduced to less than $40 \%$, and that the risk over each of the next 5 years is a tiny fraction of that, she can know great release from the pressure to make major decisions about prophylactic mastectomy, reconstruction, and the timing of the two while feeling "under the gun."

The more that major series such as this one from Rotterdam can influence the advice given to women at risk, the greater their comfort in decision making, and the greater the comfort to those providing counsel.

\section{REFERENCES}

1. Heemskerk-Gerritsen, Brekelmans, Menke-Pluijmers, et al. Prophylactic mastectomy in BRCA1/2 mutation carriers and women at risk of hereditary breast cancer: long-term experiences at the Rotterdam Family Cancer Clinic. Ann Surg Oncol 2007; 14: doi:10.1245/s10434-007-9449-x [Online May 31, 2007].

2. Kuhl C, Schrading S, Leutner CC, et al. Mammography, breast ultrasound, and magnetic resonance imaging for surveillance of women at high familial risk of breast cancer. J Clin Oncol 2005; 23:8469-8476.

3. Eisen A, Lubinski J, Klijn J, et al. Breast cancer risk following bilateral oophorectomy in BRCA1 and BRCA2 mutation carriers: an international case control study. J Clin Oncol 2005; 23:7491-7496.

4. Gronwald J, Tung N, Foulkes WD, et al. Tamoxifen and contralateral breast cancer in BRCA1 and BRCA2 carriers: an update. Int J Cancer 2006; 118:2281-2284. 